Historic, Archive Document

Do not assume content reflects current scientific knowledge, policies, or practices. 



\title{
6239
}

\section{WHOLESALE TRADE LIST FALL OF 1929}

\section{Gillett's}

Fern and Flower Farm

\author{
KENNETH E. GILLETT, Proprietor \\ SOUTHWICK, MASSACHUSETTS
}

Orders accepted under usual trade terms and conditions, boxing at cost.

All of the stock is of my own growing and is now in my nursery ready for shipment.

This list supersedes all previous catalogs and quotations.

Twenty-five of one variety will be sold at the hundred rate; five at the ten rate; where less than five of one variety are ordered add $25 \%$ to the ten rate.

\section{HARDY NATIVE FERNS}

Adiantum pedatum (Maidenhair Fern)

Aspidium acrostichoides (Christmas Fern)

Aspidium aculeatum Braunii (Prickly Shield Fern)

Aspidium cristatum (Crested Wood Fern)

Aspidium cristatum Clintonianum

Aspidium Goldieanum (Goldie's Wood Fern)

Aspidium marginale (Evergreen Wood Fern)

Aspidium noveboracense (New York Shield Fern)

Aspidium spinulosum (Spinulose Wood Fern)

Aspidium spinulosum dilatatum

Aspidium thelypteris (Marsh Shield Fern)

Asplenium angustifolium (Narrow-leaved Spleenwort)

Asplenium ebeneum (Ebony Spleenwort)

Asplenium Filix-foemina (Lady Fern)

Asplenium thelypteroides (Silvery Spleenwort)

Asplenium trichomanes (Maidenhair Spleenwort)

Cystopteris bulbifera (Bladder Fern)

Cystopteris fragilis (Fragile Fern)

Dicksonia punctilobula (Hayscented Fern) strong clumps

Dicksonia punctilobula, Sq. Ft. sods

Onoclea sensibilis (Sensitive Fern)

Onoclea struthiopteris (Ostrich Fern)

Ophioglossum vulgatum (Adder's-Tongue Fern)

Osmunda cinnamomea (Cinnamon Fern)

Osmunda Claytoniana

Osmunda regalis (Royal Fern)

Phegopteris Dryopteris (Beech Fern)

Phegopteris hexagonoptera

Per 10 Per 100

$\$ 1.00 \quad \$ 8.00$

$\begin{array}{ll}1.00 & 8.00\end{array}$

$2.30 \quad 20.00$

$1.00 \quad 8.00$

$1.00 \quad 8.00$

$3.00 \quad 25.00$

$1.00 \quad 8.00$

$1.00 \quad 8.00$

$1.00 \quad 8.00$

$1.35 \quad 10.00$

$1.00 \quad 8.00$

14.00

$\begin{array}{rr}1.00 & 8.00 \\ 1.00 & 8.00\end{array}$

$1.00 \quad 8.00$

$1.00 \quad 8.00$

$1.00 \quad 8.00$

$1.00 \quad 8.00$

$\begin{array}{ll}1.00 & 8.00\end{array}$

$2.50 \quad 20.00$

$\begin{array}{ll}1.00 & 8.00\end{array}$

$1.25 \quad 10.00$

$\begin{array}{ll}1.00 & 8.00\end{array}$

$\begin{array}{ll}1.25 & 10.00\end{array}$

$\begin{array}{ll}1.25 & 10.00\end{array}$

$1.25 \quad 10.00$

$\begin{array}{ll}1.00 & 8.00\end{array}$

$1.00 \quad 8.00$ 
Phegopteris polypodioides

Polypodium vulgare (Common Polypody)

Pteris aquilina (Common Brake)

Woodsia ilvensis (Rusty Woodsia)

Woodsia obtusa (Obtuse-leaved Woodsia)

Woodwardia virginica

\section{HARDY PERENNIALS}

Actaea alba (White Baneberry)

Per 10 Per 100

Actaea rubra (Red Baneberry)

$\$ 1.50$

Alyssum saxatile compactum

Aquilegia canadensis

Androsace primuloides

Arabis Kelleri (a miniature rock Cress, new)

Arabis mollis

Arenaria caespitosa

Arisaema triphyllum

Asarum canadense (Wild Ginger)

Asclepias tuberosa (Butterfly Weed)

Aster cordifolia

Aster ericoides

Aster laevis

Aster linariifolius

Aster multiflorus

Aster novi-belgii

Baptisia tinctoria

Caulophyllum thalictroides (Blue Cohosh)

Chelone glabra

Cimicifuga racemosa

Claytonia virginica

Clintonia borealis

Coptis trifolia

Cornus canadensis (clumps with earth)

Dianthus deltoides

Dianthus plumarius

Dicentra canadensis

Dicentra Cucullaria

Dodecatheon media

Erythronium albidum (large bulbs)

Erythronium Americanum (large bulbs)

Erythronium grandiflorum

Eupatorium ageratoides

Eupatorium perfoliatum

Eupatorium purpureum

Galax aphylla

Gaultheria procumbens (clumps with earth)

Gentiana Andrewsii

Geranium maculatum

Hepatica acutiloba (strong clumps)

Hepatica triloba

Houstonia caerulea

Hypoxis erecta

Iberis sempervirens

Iris cristata

Iris Pseudacorus

Iris pumila hybrida (blue)

Iris Snow Queen

Iris verna

Iris versicolor

Lilium canadense 1st size

Lilium philadelphicum

Lilium superbum, 1st size

Lobelia cardinalis

Lobelia syphilitica

Lupinus perennis

Lysimachia Nummularia

Lythrum roseum superbum

Mertensia virginica. 1st size ( $\$ 75$ per $M)$

Mitchella repens (clumps with earth)
1.75

1.25

1.00

Per 10 Per 100

8.00

$1.00 \quad 8.00$

8.00

$1.00-8.00$

$1.00 \quad 8.00$

$\begin{array}{rr}1.25 & 8.00\end{array}$

$2.00 \quad 15.00$

$1.00 \quad 8.00$

$1.00 \quad 8.00$

$1.00 \quad 8.00$

$1.00 \quad 8.00$

$1.25 \quad 10.00$

$1.00 \quad 8.00$

$1.00 \quad 8.00$

$\begin{array}{ll}1.00 & 8.00\end{array}$

$1.00 \quad 8.00$

$1.00 \quad 8.00$

$1.00 \quad 8.00$

$1.00 \quad 8.00$

$1.00 \quad 8.00$

$1.00 \quad 8.00$

$1.00 \quad 8.00$

$\begin{array}{ll}.75 & 6.00\end{array}$

$1.00 \quad 8.00$

$1.00 \quad 8.00$

$1.50 \quad 12.00$

$1.00 \quad 8.00$

$1.00 \quad 8.00$

$\begin{array}{ll}.75 & 6.00\end{array}$

$\begin{array}{ll}.75 & 6.00\end{array}$

$1.25 \quad 10.00$

$\begin{array}{ll}.75 & 6.00\end{array}$

$\begin{array}{ll}.75 & 6.00\end{array}$

$\begin{array}{ll}.75 & 6.00\end{array}$

$1.00 \quad 8.00$

$1.00 \quad 8.00$

$1.00 \quad 8.00$

$1.25 \quad 10.00$

$1.50 \quad 12.00$

$1.00 \quad 8.00$

$1.00 \quad 8.00$

$1.25 \quad 10.00$

$1.00 \quad 8.00$

$\begin{array}{ll}.75 & 6.00\end{array}$

$1.00 \quad 8.00$

$1.25 \quad 10.00$

$1.00 \quad 8.00$

$1.00 \quad 8.00$

$1.25 \quad 10.00$

$1.00 \quad 8.00$

$1.00 \quad 8.00$

$1.00 \quad 8.00$

$1.25 \quad 10.00$

$1.25 \quad 10.00$

$1.25 \quad 10.00$

$1.00 \quad 8.00$

$1.00 \quad 8.00$

$1.00 \quad 8.00$

$1.00 \quad 8.00$

$1.00 \quad 8.00$

$1.00 \quad 8.00$
1.25

$1.25 \quad 10.00$ 
Mitella dìphylla

Per 10 Per 100

Myosotis palustris

1.00

8.00

Parnassia carolinian

1.00

Phlox divaricata

8.00

Phlox subulata

$1.00 \quad 8.00$

Phlox subulata alba

Phlox subulata lilacina

$1.00 \quad 8.00$

$1.00 \quad 8.00$
1.00

Podophyllum peltatum

1.00

8.00

Polygala paucifolia

Polygonatum biflorum

Polygonatum majus

Sanguinaria canadensis

$1.00 \quad 8.00$

$1.00 \quad 8.00$

$1.00 \quad 8.00$

$1.00 \quad 8.00$

$1.25 \quad 10.00$

$.75 \quad 6.00$

Saxifraga virginiensis

8.00

Sedum acre

Sedum album

Sedum lydium

Sedum reflexum

Sedum sexangulare

Sedum stoloniferum

Sedum stoloniferum coccineum

Sempervivum Alberti ( $\$ 50$ per M)

Sempervivum arachnoideum ( $\$ 50$ per M)

Sempervivum globiferum ( $\$ 50$ per $M$ )

Sempervivum tectorum ( $\$ 50$ per $M)$

1.00

1.00

1.00

8.00

$1.00-8.00$

$1.00 \quad 8.00$

$1.00 \quad 8.00$

$1.00 \quad 8.00$

$1.00 \quad 8.00$

$.75 \quad 6.00$

$.75 \quad 6.00$

$.75 \quad 6.00$

$.75 \quad 6.00$

Shortia galacifolia

Smilacina bifolia

2.50

1.00

20.00

Smilacina racemosa

Solidago canadensis

Solidago memoralis

Stylophorum diphyllum

Thalictrum dioicum

Thalictrum cornuti

Thymus azoricus

Thymus lanuginosus (Wolly Thyme)

1.00

1.00

1.00

1.25

.75

1.00

1.00

1.00

1.00

Thymus serpyllum

Thymus serpyllum album

1.00

1.00

Thymus serpyllum coccineum

Tiarella cordifolia

Tradescantia virginiana.

Trillium erectum

Trillium erythrocarpum

Trillium grandiflorum ( $\$ 30$ per M)

1.00

1.00

.65

.75

Trillium stylosum

Uvularia perfoliata

1.00

Vernonia noveboracensis

Veronica prostrata

Vinca minor (field grown clumps)

1.00

1.00

1.25

Viola blanda

1.00

1.00

Viola canadensis

Viola canina sylvestris

1.00

1.00

Viola cornuta

Viola cucullata

Viola Johnny-Jump-Ups

Viola palmata

Viola pedata

Viola pubescens

1.00

8.00

8.00

8.00

8.00

10.00

6.00

8.00

8.00

8.00

8.00

8.00

8.00

8.00

8.00

5.00

6.00

4.00

8.00

8.00

8.00

8.00

10.00

8.00

8.00

8.00

8.00

8.00

6.00

8.00

8.00

8.00

8.00

Viola septentrionalis

BOG PLANTS

Acorus calamus

Calla palustris

Caltha palustris

Per 100

$\$ 1.00 \quad \$ 8.00$

$\begin{array}{ll}.75 & 6.00\end{array}$

$.75 \quad 6.00$

$.75 \quad 6.00$

Menyanthes trifoliata

1.25

10.00

$1.25 \quad 10.00$

Sarracenia rubra

8.00

1.00

6.00

Scirpus atrovirens

Vaccinium macrocarpon

.75

6.00 


\section{NATIVE ORCHIDS}

Cypripedium acaule ( 1 to 2 crowns)

Cypripedium pubescens ( 1 to 2 crowns)

Cypripedium pubescens (3 to 4 crowns)

Cypripedium spectabile ( 1 to 2 crowns)

Cypripedium spectabile ( 3 to 4 crowns)

Cypripedium spectabile ( 5 to 8 crowns)

Goodyera pubescens

Pogonia ophioglossoides

$\begin{array}{rr}\text { Per 10 } & \text { Per } 100 \\ \$ 1.25 & \$ 10.00 \\ 1.75 & 15.00 \\ 3.50 & 30.00 \\ 2.50 & 20.00 \\ 5.00 & 40.00 \\ 10.00 & \\ 1.00 & 8.00 \\ 1.00 & 8.00\end{array}$

\section{EVERGREEN SHRUBS}

Prices include balling and burlapping

Andromeda floribunda 12-15 in. spread

Andromeda floribunda 15-18 in. spread

Per 10 Per 100

$\$ 17.50 \quad \$ 150.00$

$22.50 \quad 200.00$

(My Andromedas are heavily budded)

Calluna vulgaris 8-12 in.

Calluna vulgaris $12-15$ in.

$8.50 \quad 75.00$

$10.00 \quad 90.00$

$12.50 \quad 110.00$

$\begin{array}{lll}\text { Ilex glabra (Inkberry) } 12-15 \text { in. } & 12.50 & 100.00 \\ \text { Ilex glabra 15-18 in. } & 17.50 & \end{array}$

Kalmia latifolia 12-15 in.

$8.50 \quad 75.00$

Kalmia latifolia $15-18$ in.

$12.50 \quad 100.00$

Kalmia latifolia 18-24 in.

$17.50 \quad 150.00$

Kalmia latifolia $2 \mathrm{ft}$.

Leucothoe catesbaei (Drooping Leucothoe) bushy clumps $12-15$ in.

$20.00 \quad 175.00$

Leucothoe catesbaei 15-18 in.

$6.50 \quad 50.00$

$8.50 \quad 75.00$

$1.25 \quad 10.00$

Pachysandra terminalis $2 \mathrm{yr}$.

All Rhododendrons in nursery grown grades have been grown from stock cut back to the ground; all new tops; bushy, with fine foliage.

Rhododendron carolinianum 12-15 in.

$\$ 12.50 \quad \$ 100.00$

Rhododendron carolinianum 15-18 in

$16.50 \quad 145.00$

$\begin{array}{lll}\text { Rhododendron carolinianum } 18 \mathrm{in} \text {. selected } & 22.50 & 200.00 \\ \text { Rhododendron catawbiense } 1-11 / 2 \mathrm{ft} . & 12.50 & \end{array}$

Rhododendron maximum 12-15 in. $\quad 10.00$

Rhododendron maximum 15-18 in. $\quad 12.50$

85.00

$12.50 \quad 100.00$

Rhododendron maximum 18-24 in. $\quad 16.00 \quad 140.00$

\section{DECIDUOUS SHRUBS}

The prices on A.zaleas include balling and burlapping

Azalea calendulacea $12-15$ in.

Azalea calendulacea $15-18$ in.

Azalea calendulacea 18-24 in.

Per 10 Per 100

$\$ 10.00 \$ 85.00$

$12.50 \quad 100.00$

$16.50 \quad 150.00$

Azalea canescens 12-15 in.

$10.00 \quad 85.00$

Azalea canescens 15-18 in.

$12.50 \quad 100.00$

Azalea canescens 18-24 in.

Azalea nudiflora 15-18 in.

$16.50 \quad 150.00$

$10.00 \quad 85.00$

$10.00 \quad 85.00$

$12.50 \quad 100.00$

Azalea viscosa $2-3 \mathrm{ft}$.

$17.50 \quad 150.00$

A zalea viscosa $3 \mathrm{ft}$. selected

My Azaleas are all well budded.

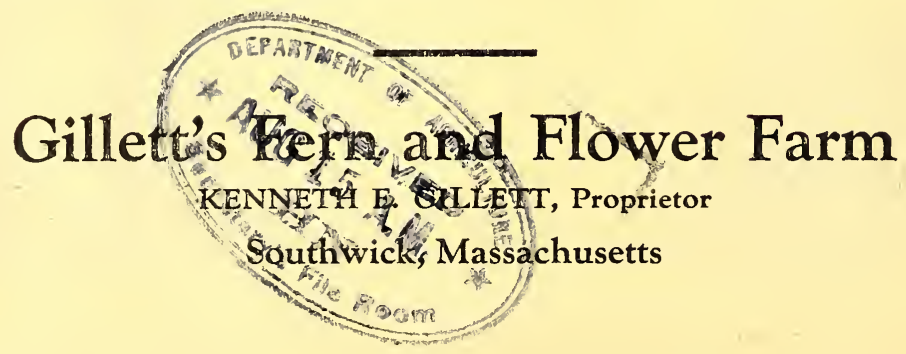

